\title{
Global hybrid multiconfiguration pair-density functional theory
}

\author{
Mohammad Mostafanejad, ${ }^{1}$ Marcus Dante Liebenthal, ${ }^{2,1}$ and A. Eugene DePrince III ${ }^{1}$ \\ 1 Department of Chemistry and Biochemistry, \\ Florida State University, Tallahassee, FL 32306-4390 \\ ${ }^{2}$ Department of Chemistry and Biochemistry, Ithaca College, Ithaca, NY 14850
}

\begin{abstract}
A global hybrid extension of variational two-electron reduced-density matrix (v2RDM)-driven multiconfiguration pair-density functional theory (MCPDFT) is developed. Using a linear decomposition of the electron-electron repulsion term, a fraction $\lambda$ of the nonlocal exchange interaction, obtained from v2RDM-driven complete active-space self-consistent field (CASSCF) theory, is combined with its local counterpart, obtained from an on-top pair-density functional. The resulting scheme (called $\lambda$-MCPDFT) inherits the benefits of MCPDFT (e.g., its simplicity and the resolution of the symmetry dilemma), and, when combined with the v2RDM approach to CASSCF, $\lambda$-MCPDFT requires only polynomially scaling computational effort. As a result, it can efficiently describe static and dynamical correlation effects in strongly correlated systems. The efficacy of the approach is assessed for several challenging multiconfigurational problems, including the dissociation of molecular nitrogen, the double dissociation of a water molecule, and the 1,3-dipolar cycloadditions of ozone to ethylene and ozone to acetylene in the O3ADD6 benchmark set.
\end{abstract}




\section{INTRODUCTION}

The search for a universally accurate and efficient delineation of electron correlation effects is an active area of research in modern electronic structure theory. [1, 2] For strongly correlated systems, in particular, where the multiconfigurational character of the wave function cannot be ignored, the single-determinental form of Kohn-Sham density functional theory (DFT) will fail, often dramatically so. In such cases, a natural remedy is to combine multireference and DFT methods (MR+DFT) to separately model strong and weak correlation effects, respectively. [3] The development of multiconfiguration pair-density functional theory (MCPDFT) [4] represents an important step in this direction, as this approach effectively capitalizes on the complementary strengths of complete active-space self-consistent field (CASSCF) theory [5-8] and DFT to offer a robust description of nondynamical correlation effects and an ecomonical representation of dynamical correlation. Moreover, MCPDFT resolves Kohn-Sham DFT's symmetry dilemma by relying on functionals of the total density and the on-top pair-density (OTPD) [4, 9, 10] (as opposed to functionals of the spin-density), and the approach avoids double counting of electron correlation within the active space. Recently, we presented [11] a reduced-density matrix (RDM)-based formulation of MCPDFT that retains these nice properties while significantly reducing the computational cost of the underlying CASSCF calculations. As such, variational two-electron RDM (v2RDM) driven MCPDFT can be applied to challenging multireference problems that require the consideration of large active spaces.

Because commonly used[4, 10] OTPD functionals and their conventional Kohn-Sham DFT counterparts only differ only in the input densities (as opposed to their actual functional forms), the MCPDFT suffers from the same "overbinding tendency" or delocalization error [12] exhibited by familiar local spin-density approximation (LSDA) and generalized gradient

approximation (GGA) exchange-correlation (XC) functionals.[13 17] This overbinding error arises because the electron-gas model [18] leads to an incorrect picture of the noninteracting limit, in which the Coulomb repulsion coupling strength is zero. The global hybrid scheme offers the simplest remedy for delocalization error by replacing a fraction of the electrongas exchange with its exact (Hartree-Fock) counterpart, which then leads to the correct noninteracting limit.[13-17]

In light of the great success of the global hybrid scheme in standard Kohn-Sham DFT, 
several proposals have been offered that extend this approach to the multiconfiguration regime 19 24]; for a survey of these techniques, the reader is referred to Ref. 3. The present study is inspired by the multiconfiguration one-parameter hybrid density functional theory $(\mathrm{MC1H})$ method of Sharkas et al.[20], which is similar to MCPDFT in that it captures nondynamical correlation with multiconfiguration self-consistent field (MCSCF) theory and the remaining dynamical correlation effects via DFT. The MC1H method has motivated the development of other MR+DFT techniques, such as pair coupled-cluster doubles $\lambda$ DFT (pCCD- $\lambda$ DFT) [21, 22] and $\lambda$-density functional valence bond $(\lambda$-DFVB), [23] where $\lambda$ refers to the hybrid parameter that controls the admixture of nonlocal exchange effects. Through this parameter, both pCCD- $\lambda$ DFT and $\lambda$-DFVB can interpolate between DFT and a MR scheme (i.e., pCCD or VB). [19, 24] In this work, we follow the general strategy proposed in Refs. 20 and 25 within the context of the v2RDM-driven formulation of MCPDFT, and we term the resulting approach $\lambda$-MCPDFT. $\lambda$-MCPDFT differs from the MC1H approach that inspired it in two respects. First, $\lambda$-MCPDFT is not a self-consistent theory. Second, like pCCD- $\lambda$ DFT (and unlike MC1H), $\lambda$-MCPDFT relies on XC functionals of the total and on-top pair densities, rather than the total and spin densities.

The manuscript is organized as follows. Section II provides the theoretical details of multiconfiguration one-parameter hybrid pair-density functional theory $(\lambda$-MCPDFT). In Sec. III, we discuss the application of $\lambda$-MCPDFT to the dissociation of molecular nitrogen and the symmetric double dissociation of a water molecule, as well as to the standard O3ADD6 benchmark set. Concluding remarks and potential future directions are then provided in Sec. IV.

\section{THEORY}

Throughout this work, we use the conventional notation of MR methods when labeling the orbitals: the indices $i, j, k$, and $l$ denote inactive (doubly occupied) orbitals; $t, u, v$, and $w$ represent active orbitals; and $p, q, r$, and $s$ indicate general orbitals. A summation over repeated indices is implied in all expressions.

We begin by defining the non-relativistic Born-Oppenheimer electronic Hamiltonian

$$
\hat{H}=h_{q}^{p} \hat{a}_{p_{\sigma}}^{\dagger} \hat{a}_{q_{\sigma}}+\frac{1}{2} \nu_{r s}^{p q} \hat{a}_{p_{\sigma}}^{\dagger} \hat{a}_{q_{\tau}}^{\dagger} \hat{a}_{s_{\tau}} \hat{a}_{r_{\sigma}}
$$


where $\hat{a}^{\dagger}$ and $\hat{a}$ represent second-quantized creation and annihilation operators, respectively, and the Greek labels run over $\alpha$ and $\beta$ spins (the sum over which is implied). The symbol $h_{q}^{p}=\left\langle\psi_{p}|\hat{h}| \psi_{q}\right\rangle$ represents the sum of the electron kinetic energy and electron-nucleus potential energy integrals, and $\nu_{r s}^{p q}=\left\langle\psi_{p} \psi_{q} \mid \psi_{r} \psi_{s}\right\rangle$ is an element of the two-electron repulsion integral tensor. Because the electronic Hamiltonian includes up to only pair-wise interactions, the ground-state energy of a many-electron system can be expressed as an exact linear functional of the the one-electron RDM (1-RDM) and two-electron RDM (2-RDM) [26-28]

$$
E={ }^{1} D_{q}^{p} h_{q}^{p}+\frac{1}{2}{ }^{2} D_{r s}^{p q} \nu_{r s}^{p q}
$$

Here, the 1-RDM and the 2-RDM are represented in their spin-free forms, with elements defined as

$$
{ }^{1} D_{q}^{p}={ }^{1} D_{q_{\sigma}}^{p_{\sigma}}=\left\langle\Psi\left|\hat{a}_{p_{\sigma}}^{\dagger} \hat{a}_{q_{\sigma}}\right| \Psi\right\rangle
$$

and

$$
{ }^{2} D_{r s}^{p q}={ }^{2} D_{r_{\sigma} s_{\tau}}^{p_{\sigma} q_{\tau}}=\left\langle\Psi\left|\hat{a}_{p_{\sigma}}^{\dagger} \hat{a}_{q_{\tau}}^{\dagger} \hat{a}_{s_{\tau}} \hat{a}_{r_{\sigma}}\right| \Psi\right\rangle
$$

respectively. Again, the summation over the spin labels in Eqs. 3 and 4 is implied.

The MCPDFT expression for the electronic energy is

$$
\begin{aligned}
E_{\mathrm{MCPDFT}} & =2 h_{i}^{i}+h_{u}^{t 1} D_{u}^{t}+E_{\mathrm{H}} \\
& +E_{\mathrm{xc}}[\rho, \Pi,|\nabla \rho|,|\nabla \Pi|],
\end{aligned}
$$

where $E_{\mathrm{xc}}$ is the translated (t)[4] or fully-translated (ft) [29] OTPD XC functional, and the Hartree energy, $E_{\mathrm{H}}$, is

$$
E_{\mathrm{H}}=2 \nu_{i j}^{i j}+2 \nu_{u i}^{t i 1} D_{u}^{t}+\frac{1}{2} \nu_{u w}^{t v}{ }^{1} D_{u}^{t 1} D_{w}^{v}
$$

The total electronic density and its gradient that enter $E_{\mathrm{xc}}$ are defined as

$$
\rho(\mathbf{r})={ }^{1} D_{q}^{p} \psi_{p}^{*}(\mathbf{r}) \psi_{q}(\mathbf{r})
$$

and

$$
\nabla \rho(\mathbf{r})={ }^{1} D_{q}^{p}\left[\nabla \psi_{p}^{*}(\mathbf{r}) \psi_{q}(\mathbf{r})+\psi_{p}^{*}(\mathbf{r}) \nabla \psi_{q}(\mathbf{r})\right],
$$

respectively. The OTPD and its gradient can similarly be expressed in terms of the 2-RDM as

$$
\Pi(\mathbf{r})={ }^{2} D_{r s}^{p q} \psi_{p}^{*}(\mathbf{r}) \psi_{q}^{*}(\mathbf{r}) \psi_{r}(\mathbf{r}) \psi_{s}(\mathbf{r})
$$


and

$$
\begin{aligned}
\nabla \Pi(\mathbf{r})={ }^{2} D_{r s}^{p q} & {\left[\nabla \psi_{p}^{*}(\mathbf{r}) \psi_{q}^{*}(\mathbf{r}) \psi_{r}(\mathbf{r}) \psi_{s}(\mathbf{r})\right.} \\
& +\psi_{p}^{*}(\mathbf{r}) \nabla \psi_{q}^{*}(\mathbf{r}) \psi_{r}(\mathbf{r}) \psi_{s}(\mathbf{r}) \\
& +\psi_{p}^{*}(\mathbf{r}) \psi_{q}^{*}(\mathbf{r}) \nabla \psi_{r}(\mathbf{r}) \psi_{s}(\mathbf{r}) \\
& \left.+\psi_{p}^{*}(\mathbf{r}) \psi_{q}^{*}(\mathbf{r}) \psi_{r}(\mathbf{r}) \nabla \psi_{s}(\mathbf{r})\right]
\end{aligned}
$$

respectively. In the MCPDFT formalism, the 1-RDM and 2-RDM can be taken from any reference calculation capable of generating "good" RDMs that include nondynamical correlation effects. In this work, the 1-RDM and 2-RDM are taken from v2RDM-driven CASSCF calculations, and they satisfy either two-particle (PQG)[30] or PQG plus partial threeparticle (T2) [31, 32] $N$-representability conditions.

In order to reduce delocalization error in the MCPDFT formalism, we define the $\lambda$-MCPDFT energy expression in which a fraction $\lambda \in(0,1)$ of the OTPD exchange is replaced with contributions from the reference 1- and 2-RDM:

$$
\begin{aligned}
E_{\lambda-\mathrm{MCPDFT}} & =2 h_{i}^{i}+h_{u}^{t 1} D_{u}^{t}+E_{\mathrm{H}} \\
& +\lambda\left(\frac{1}{2} \nu_{u w}^{t v}\left[{ }^{2} \Delta_{u w}^{t v}-{ }^{1} D_{w}^{t 1} D_{u}^{v}\right]-\nu_{i i}^{t u 1} D_{u}^{t}-\nu_{j j}^{i i}\right) \\
& +\bar{E}_{\mathrm{xc}}^{\lambda}[\rho, \Pi,|\nabla \rho|,|\nabla \Pi|],
\end{aligned}
$$

Here, the two-cumulant, ${ }^{2} \Delta$, is the part of the 2 -RDM that cannot be represented in terms of the $1-\mathrm{RDM}$ and is defined according to

$$
{ }^{2} D_{u w}^{t v}={ }^{2} \Delta_{u w}^{t v}+{ }^{1} D_{u}^{t 1} D_{w}^{v}-{ }^{1} D_{w}^{t 1} D_{u}^{v}
$$

The complement OTPD functional, $\bar{E}_{\text {xc }}^{\lambda}[\cdot]$, is given by

$$
\begin{aligned}
\bar{E}_{\mathrm{xc}}^{\lambda}[\rho, \Pi,|\nabla \rho|,|\nabla \Pi|] & =E_{\mathrm{xc}}[\rho, \Pi,|\nabla \rho|,|\nabla \Pi|] \\
& -E_{\mathrm{xc}}^{\lambda}[\rho, \Pi,|\nabla \rho|,|\nabla \Pi|]
\end{aligned}
$$

which is the difference between the conventional exchange-correlation OTPD functional $\left(E_{\mathrm{xc}}[\cdot]\right)$ and its $\lambda$-dependent hybrid version $\left(E_{\mathrm{xc}}^{\lambda}[\cdot]\right) \cdot[20]$

The exchange part of the complement functional is easily defined, as it simply scales linearly with the mixing parameter $\lambda[19,20,25]$

$$
\bar{E}_{\mathrm{x}}^{\lambda}[\rho, \Pi,|\nabla \rho|,|\nabla \Pi|]=(1-\lambda) E_{\mathrm{x}}[\rho, \Pi,|\nabla \rho|,|\nabla \Pi|]
$$


The correlation contribution to the complement OTPD functional can be obtained through uniform coordinate scaling of the density as [19, 20, 25]

$$
\begin{aligned}
& \bar{E}_{\mathrm{c}}^{\lambda}[\rho, \Pi,|\nabla \rho|,|\nabla \Pi|]=E_{\mathrm{c}}[\rho, \Pi,|\nabla \rho|,|\nabla \Pi|]-E_{\mathrm{c}}^{\lambda}[\rho, \Pi,|\nabla \rho|,|\nabla \Pi|] \\
& =E_{\mathrm{c}}[\rho, \Pi,|\nabla \rho|,|\nabla \Pi|]-\lambda^{2} E_{\mathrm{c}}\left[\rho^{1 / \lambda}, \Pi^{1 / \lambda},\left|\nabla \rho^{1 / \lambda}\right|,\left|\nabla \Pi^{1 / \lambda}\right|\right]
\end{aligned}
$$

in which the scaled density and OTPD functions are defined as [33]

$$
\begin{aligned}
\rho^{1 / \lambda}(\mathbf{r}) & =\left(1 / \lambda^{3}\right) \rho(\mathbf{r} / \lambda) \\
\Pi^{1 / \lambda}(\mathbf{r}) & =\left(1 / \lambda^{6}\right) \Pi(\mathbf{r} / \lambda)
\end{aligned}
$$

Following Ref. [20], we neglect the scaling relations of the total density and OTPD in the correlation complement functional and define

$$
\bar{E}_{\mathrm{c}}^{\lambda}[\rho, \Pi,|\nabla \rho|,|\nabla \Pi|]=\left(1-\lambda^{2}\right) E_{\mathrm{c}}[\rho, \Pi,|\nabla \rho|,|\nabla \Pi|]
$$

Using Eqs. 14 and 18, the $\lambda$-MCPDFT energy expression takes its final form:

$$
\begin{aligned}
E_{\lambda-\mathrm{MCPDFT}} & =2 h_{i}^{i}+h_{u}^{t 1} D_{u}^{t}+E_{\mathrm{H}} \\
& +\lambda\left(\frac{1}{2} \nu_{u w}^{t v}\left[{ }^{2} \Delta_{u w}^{t v}-{ }^{1} D_{w}^{t}{ }^{1} D_{u}^{v}\right]-\nu_{i i}^{t u 1} D_{u}^{t}-\nu_{j j}^{i i}\right) \\
& +(1-\lambda) E_{\mathrm{x}}[\rho, \Pi,|\nabla \rho|,|\nabla \Pi|] \\
& +\left(1-\lambda^{2}\right) E_{\mathrm{c}}[\rho, \Pi,|\nabla \rho|,|\nabla \Pi|]
\end{aligned}
$$

where, for $\lambda=0$ or $\lambda=1$, the $\lambda$-MCPDFT energy expression reduces to that corresponding to MCPDFT or the underlying multiconfiguration reference method, respectively.

\section{RESULTS AND DISCUSSION}

The RDMs that enter the MCPDFT and $\lambda$-MCPDFT equations were obtained from v2RDM-driven CASSCF calculations performed using a plugin [34] to the PSI4 electronic structure package; [35] the optimized RDMs satisfy either the PQG or PQG+T2 Nrepresentability conditions. Both the MCPDFT and $\lambda$-MCPDFT procedures, along with the translated and fully-translated versions of the SVWN3, [36 38] PBE [39], and BLYP [40, 41] XC functionals, have also been implemented in our open-source library (OPENRDM) [42] which is interfaced as a plugin to Psi4.[43] 


\section{A. $\mathrm{N}_{2}$ and $\mathrm{H}_{2} \mathrm{O}$ Bond Dissociations}

In this section, we consider the accuracy of MCPDFT and $\lambda$-MCPDFT for describing the dissociation of $\mathrm{N}_{2}$ and the double dissociation of $\mathrm{H}_{2} \mathrm{O}$; the non-parallelity errors (NPEs) in the respective potential energy curves (PECs), which are obtained by comparing the MCPDFT and $\lambda$-MCPDFT results with those from complete active-space second-order perturbation theory (CASPT2), serve as a useful metric in this context. The CASSCF calculations underlying CASPT2, MCPDFT, and $\lambda$-MCPDFT employed full-valence active

spaces and the cc-pVTZ basis set, [44] and, in the case of v2RDM-driven CASSCF, the electron repulsion integral (ERI) tensor was represented within the density-fitting (DF) approximation, [45, 46] using the cc-pVTZ-JK auxiliary basis set.[47] All MCPDFT and $\lambda$-MCPDFT computations also employed the DF approximation. The CASPT2 computations were performed using the OPEN-MOLCAS electronic structure package, [48] and the standard imaginary shift [49] of $0.20 \mathrm{E}_{\mathrm{h}}$ and OPEN-MOLCAS's default value of $0.25 \mathrm{E}_{\mathrm{h}}$ for the IPEA shift[50] were applied throughout.

Figure 1 illustrates the effect of the mixing parameter, $\lambda$, on the NPE for $\mathrm{N}_{2}$ when enforcing the PQG or PQG+T2 N-representability conditions and adopting either translated or fully-translated $\lambda$-MCPDFT functionals. The NPE is defined as the difference in the maximum and the minimum deviations between the $\lambda$-MCPDFT and CASPT2 PECs from an $\mathrm{N}-\mathrm{N}$ distance of $0.7 \AA$ to an $\mathrm{N}-\mathrm{N}$ distance of $5.0 \AA$. With the exception of that for tBLYP combined with the PQG $N$-representability conditions, all NPE curves presented in Fig. 1] exhibit their minimal value between $\lambda=0.70$ and $\lambda=0.90$. Specifically, when enforcing the PQG (PQG+T2) N-representability conditions and adopting tSVWN3, tPBE, tBLYP, ftSVWN3, ftPBE, and ftBLYP functionals, the optimal $\lambda$ values are 0.90 (0.90), 0.00 (0.80), 0.75 (0.80), 0.90 (0.90), 0.80 (0.80), and 0.80 (0.80), respectively. For tPBE/PQG, it appears that the optimal $\lambda$ value is 0.00 in this case, although the NPE at $\lambda=0.80$ is only $0.3 \mathrm{kcal}$ $\mathrm{mol}^{-1}$ larger. Note that the optimal values of the mixing parameters in all other cases lie between $\lambda=0.70$ and $\lambda=0.90$, which are close to the value of $\lambda=0.75$ used within the pCCD- $\lambda$ DFT-based study of $\mathrm{N}_{2}$ dissociation presented in Ref. 22 .

Figure 2 presents the NPEs associated with the MCPDFT and hybrid $\lambda$-MCPDFT PECs, using the optimal $\lambda$ values identified for each functional / $N$-representability combination considered in Fig. 1, and the data reveal several interesting features. First, as noted in 
FIG. 1: The NPE in the dissociation curves for the $\mathrm{N}_{2}$ molecule as a function of the mixing parameter, $\lambda$, when using PQG (a),(b) or PQG+T2 (c),(d) $N$-representability conditions and translated (a),(c) or fully-translated (b),(d) $\lambda$-MCPDFT functionals.
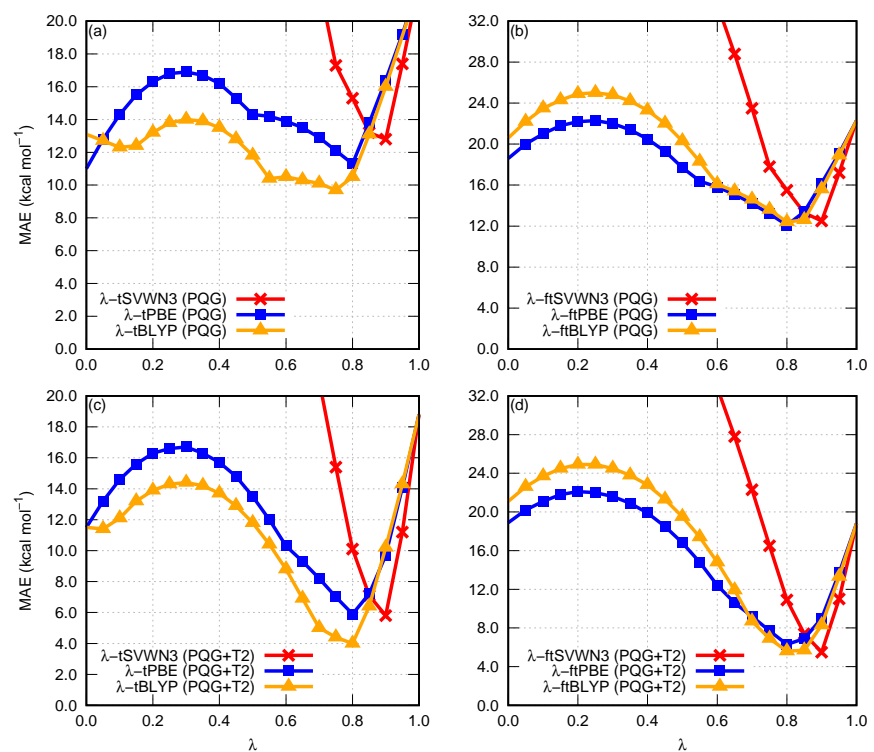

Ref. 11, for both translated and fully-translated MCPDFT functionals, the NPE is somewhat insensitive to the $N$-representability of the RDMs. On the other hand, $\lambda$-MCPDFT clearly yields lower NPE values when the underlying RDMs satisfy both the PQG and T2 conditions; it appears that the consideration of nonlocal exchange effects alleviates enough of the delocalization error to allow us to discern the error associated with approximate $N$ representability of the reference RDMs. Second, while the NPEs vary significantly with the choice of MCPDFT functional, nonlocal exchange effects serve as a great equalizer; the NPE are quite similar for all $\lambda$-MCPDFT methods, when enforcing a given set of $N$ representability conditions. Unsurprisingly, the improvement in the NPEs is largest for the $\lambda$-tSVWN3 and $\lambda$-ftSVWN3 functionals. Third, as observed in Ref. 11, the choice of translated or fully-translated functionals does not significantly affect the NPE associated with functionals within either the MCPDFT or $\lambda$-MCPDFT formalisms. Fully-translated functionals yield slightly lower NPE values than translated functionals, but this difference is far less significant than the improvements afforded by the admixture of nonlocal exchange effects.

Similar trends are observed for the NPEs associated with the $\mathrm{H}_{2} \mathrm{O}$ double-dissociation PEC. Figure 3 depicts the NPE for each functional, where the mixing parameter is chosen to minimize the $\lambda$-MCPDFT NPE between $\mathrm{O}-\mathrm{H}$ bond distances of $0.6 \AA$ and $5.0 \AA$. In 
FIG. 2: MCPDFT and $\lambda$-MCPDFT NPEs (mhartree) associated with the dissocation of $\mathrm{N}_{2}$. The mixing parameter for $\lambda$-MCPDFT is chosen to be that which gives the lowest NPE for each functional considered in Fig. 1, Each concentric ring denotes an additional 15 mhartree error.

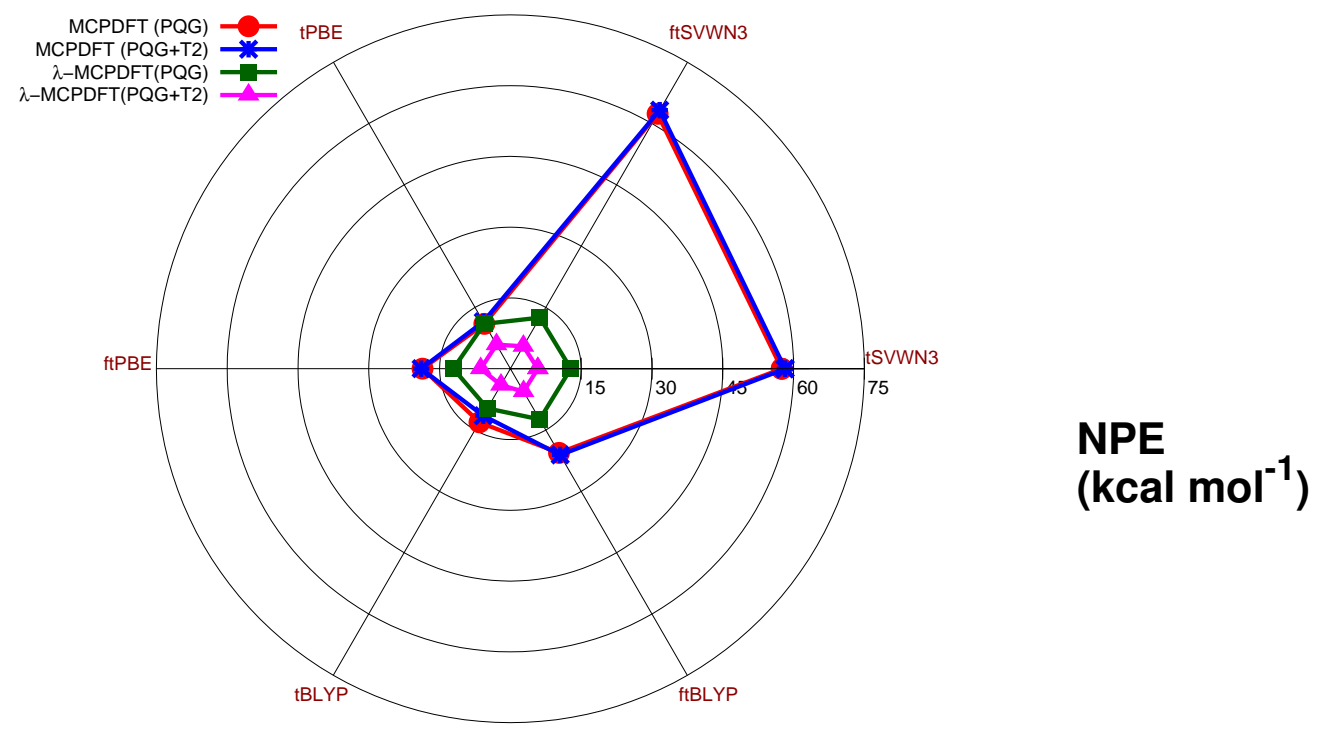

this case, when enforcing the PQG (PQG+T2) $N$-representability conditions and adopting tSVWN3, tPBE, tBLYP, ftSVWN3, ftPBE, and ftBLYP functionals, the optimal $\lambda$ values are $0.70(0.70), 0.45(0.50), 0.45$ (0.50), 0.70 (0.70), 0.50 (0.50), and 0.50 (0.50), respectively. Relative to the case of $\mathrm{N}_{2}$, the optimal mixing parameters, in general, are smaller for this problem. The only exception is tPBE, where the optimal value of $\lambda=0.45$ is clearly larger than the optimal value for $\mathrm{N}_{2}$ dissociation (0.0). We again note that the inclusion of nonlocal exchange effects significantly reduces the NPEs, in general, and the variation in the performance of $\lambda$-MCPDFT functionals is much less than that of the MCPDFT functionals. In constrast to the case of $\mathrm{N}_{2}$, the quality of the NPEs associated with $\lambda$-MCPDFT functionals is less dependent upon the $N$-representability of the underying RDMs. The NPEs for each $\lambda$-MCPDFT functional, as a function of the mixing parameter, are depicted in Fig. S1 of the Supporting Information.

\section{B. O3ADD6 Benchmark Set}

The O3ADD6 benchmark set is comprised of the energies of three stationary points associated with the 1,3-dipolar cycloadditions of ozone $\left(\mathrm{O}_{3}\right)$ to ethylene $\left(\mathrm{C}_{2} \mathrm{H}_{4}\right)$ and $\mathrm{O}_{3}$ to acetylene $\left(\mathrm{C}_{2} \mathrm{H}_{2}\right)$, relative to the energies of the isolated reactants. The stationary points [the 
FIG. 3: MCPDFT and $\lambda$-MCPDFT NPEs (mhartree) associated with the double dissociation of $\mathrm{H}_{2} \mathrm{O}$. The mixing parameter for $\lambda$-MCPDFT is chosen to be that which gives the lowest NPE for each functional (see Fig. S1 of the Supporting Information). Each concentric ring denotes an additional 15 mhartree error.

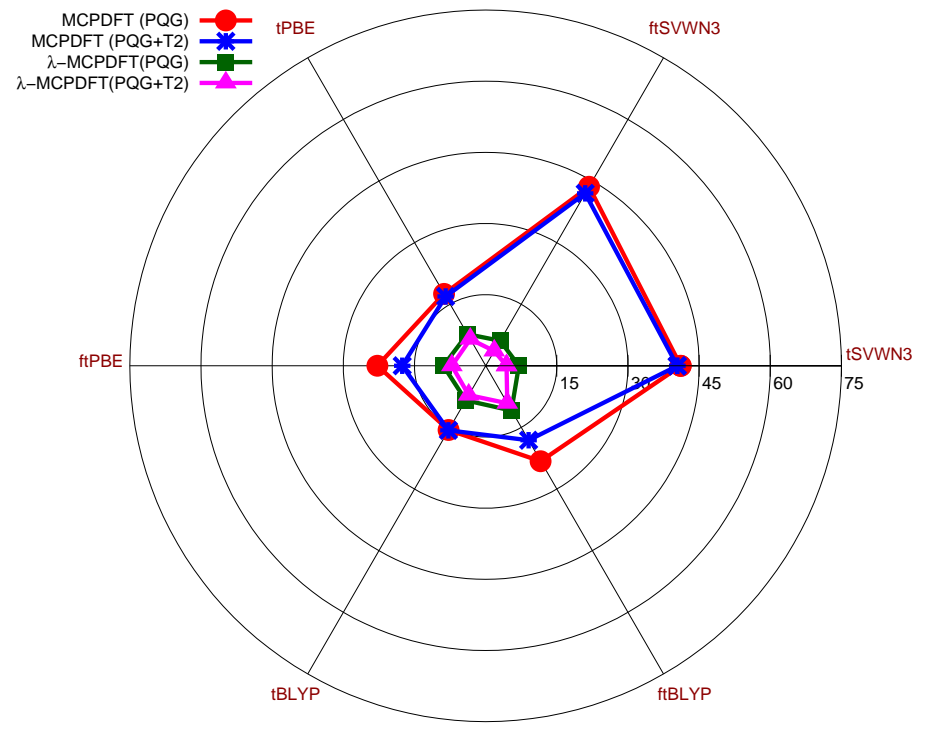

\section{NPE (kcal mol ${ }^{-1}$ )}

van der Waals complex (vdW), the transition state (TS), and the cycloadduct (Cycloadd.)] and the separated reactants are all assumed to be in singlet spin states. These systems present a challenge to quantum chemical methods, as the highest-occupied molecular orbital $(\mathrm{HOMO}) \rightarrow$ lowest-unoccupied molecular orbital (LUMO) double excitation character in $\mathrm{O}_{3}$ and the near-degeneracy of the $\pi$ and $\pi^{*}$ orbitals corresponding to the reactive $\pi$ bonds in the olefins result in strong MR character in several of the species along the reaction coordinate. [20, 51] Here, we apply the MCPDFT and $\lambda$-MCPDFT methods to this data set, using the aug-cc-pVTZ basis set[44] and geometries for the stationary points given in Ref. 52. All v2RDM-driven CASSCF and $\lambda$-MCPDFT calculations employed the DF approximation to the ERI tensor and the aug-cc-pVTZ-JK auxiliary basis set. In order to compare our results with those from Refs. 20 and 52 , we consider an active space comprised of 2 electrons in 2 orbitals [denoted as $(2 \mathrm{e}, 2 \mathrm{o})]$ for the reactants and $(4 \mathrm{e}, 4 \mathrm{o})$ active spaces for the vdW, TS, and Cycloadd. species; the orbitals comprising each active space are defined in Ref. 20. Following Refs. 52 and 20, we neglect vibrational zero-point energy contributions to the energies of the stationary points and separated reactants.

We identify the optimal nonlocal exchange mixing parameter, specific to the O3ADD6 benchmark set, for several $\lambda$-MCPDFT functionals by minimizing the mean absolute error 
(MAE) in the relative energies of the stationary points and the isolated reactant molecules. The reference values to which the calculated relative energies are compared are taken from Ref.52. Fig. 4depicts the MAEs in the calculated relative energies as a function of $\lambda$, and the data clearly convey the importance of nonlocal exchange effects for this problem, particularly in the case of $\lambda$-tSVWN3 and $\lambda$-ftSVWN3. For all functionals considered, the inclusion of some fraction of nonlocal exchange is beneficial, although this fraction is, in general, smaller than the optimal fraction for minimizing the NPE in the $\mathrm{N}_{2}$ and $\mathrm{H}_{2} \mathrm{O}$ dissociation curves considered above. The optimal $\lambda$ values, which are tabulated in Table【and in Table S1 in the Supporting Information, are insensitive to the choice of $N$-represetability conditions. The only functional that requires a different mixing parameter under different $N$-representability conditions is tSVWN3, and this value only changes by 0.05 in this case. One possible reason for this insensitivity is the small size of the active spaces considered. For example, for the $(2 \mathrm{e}, 2 \mathrm{o})$ active space, the two-particle conditions alone are sufficient to yield ensemble-state $N$-representable RDMs.

FIG. 4: The MAE ( $\mathrm{kcal} \mathrm{mol}^{-1}$ ) in calculated O3ADD6 energies as a function of the mixing parameter, $\lambda$, when RDMs satisfy PQG (a),(b) and PQG+T2 (c),(d) $N$-representability conditions and when adopting translated (a),(c) and fully-translated (b),(d) $\lambda$-MCPDFT functionals.
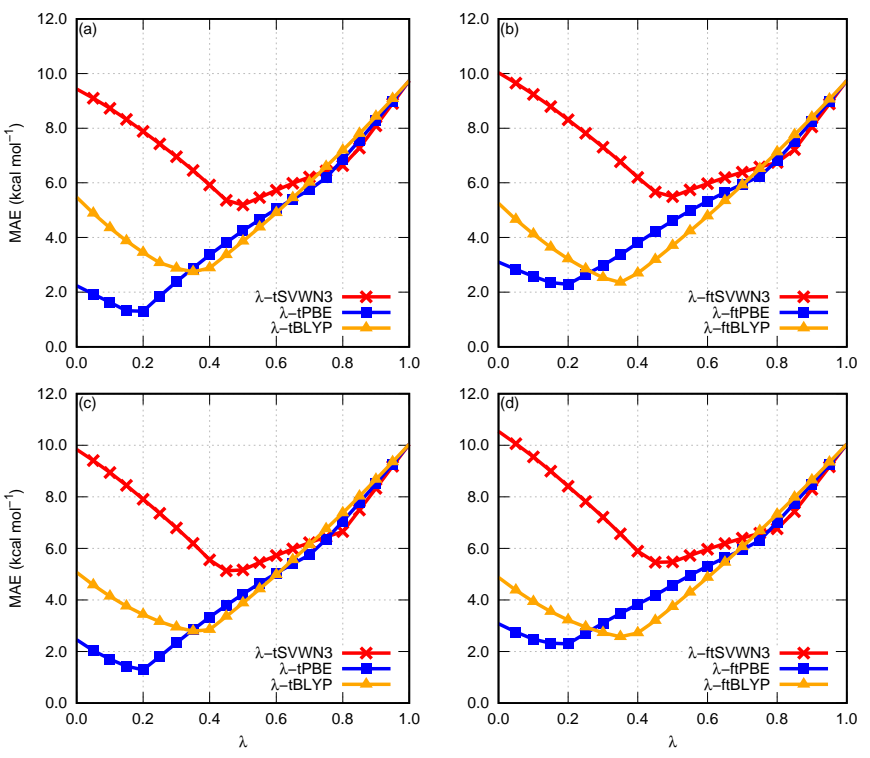

While the v2RDM-driven CASSCF method can capture nondynamical correlation effects that are important in the systems comprising the O3ADD6 set, the large average errors associated with the approach $\left(9.73\right.$ and $10.04 \mathrm{kcal} \mathrm{mol}^{-1}$ when the calculations are performed 
TABLE I: Calculated relative energies $\left(\mathrm{kcal} \mathrm{mol}^{-1}\right)$ of the stationary points and separated reactant molecules that comprise the O3ADD6 dataset.

\begin{tabular}{|c|c|c|c|c|c|c|c|c|c|}
\hline \multirow{2}{*}{ Method } & \multirow{2}{*}{$N$-representability } & \multirow{2}{*}{$\lambda^{a}$} & \multicolumn{3}{|c|}{$\mathrm{O}_{3}+\mathrm{C}_{2} \mathrm{H}_{2} \rightarrow$} & \multicolumn{3}{|c|}{$\mathrm{O}_{3}+\mathrm{C}_{2} \mathrm{H}_{4} \rightarrow$} & \multirow{2}{*}{ - MAE } \\
\hline & & & $\mathrm{vdW}$ & TS & Cycloadduct & $\mathrm{vdW}$ & TS & Cycloadduct & \\
\hline$\lambda$-tPBE & & 0.20 & -0.42 & 7.84 & -67.75 & -2.19 & 4.91 & -57.66 & 1.30 \\
\hline$\lambda$-tBLYP & & 0.35 & 0.24 & 12.51 & -61.41 & -2.70 & 8.82 & -56.18 & 2.75 \\
\hline$\lambda$-tSVWN3 & PQG & 0.50 & -1.15 & 7.72 & -73.88 & -5.72 & 4.69 & -72.34 & 5.19 \\
\hline v2RDM-CASSCF & & 1.00 & 0.55 & 23.33 & -56.41 & -6.93 & 18.97 & -69.54 & 9.73 \\
\hline$\lambda$-tPBE & & 0.20 & -0.40 & 7.69 & -68.00 & -1.86 & 4.87 & -57.57 & 1.29 \\
\hline$\lambda$-tBLYP & & 0.35 & 0.27 & 12.82 & -61.51 & -1.97 & 9.23 & -55.88 & 2.78 \\
\hline$\lambda$-tSVWN3 & $\mathrm{PQG}+\mathrm{T} 2$ & 0.45 & -1.26 & 6.92 & -75.19 & -4.51 & 4.10 & -71.78 & 5.13 \\
\hline v2RDM-CASSCF & & 1.00 & 0.63 & 25.86 & -55.60 & -4.19 & 21.55 & -68.13 & 10.04 \\
\hline $\mathrm{MC} 1 \mathrm{H}-\mathrm{PBE}^{b}$ & & 0.25 & -1.08 & 3.66 & -70.97 & -1.25 & 0.13 & -61.26 & 3.35 \\
\hline MC1H-BLYP ${ }^{b}$ & & 0.25 & -0.36 & 6.74 & -63.76 & -0.47 & 2.57 & -54.21 & 1.30 \\
\hline Reference values $^{c}$ & & - & -1.90 & 7.74 & -63.80 & -1.94 & 3.37 & -57.15 & - \\
\hline
\end{tabular}

under the PQG and PQG+T2 N-representability conditions, respectively) reflect its inability to describe dynamical electron correlation. These effects are well described by MCPDFT and $\lambda$-MCPDFT; each hybrid functional considered here, as well as the base MCPDFT functionals considered in the Supporting Information (with the exception of the translated and fully-translated SVWN3 functionals), substantially reduce the error associated with CASSCF. In particular, the admixture of $20 \%$ nonlocal exchange into the translated PBE OTPD functional reduces the MAE to only $1.30 \mathrm{kcal} \mathrm{mol}^{-1}$ (PQG) or $1.29 \mathrm{kcal} \mathrm{mol}^{-1}$ (PQG+T2), which is close to the $1.0 \mathrm{kcal} \mathrm{mol}^{-1}$ threshold for "chemical accuracy." The description of the O3ADD6 afforded by $\lambda$-MCPDFT with this functional is similar in quality to that exhibited by the MC1H-BLYP approach of Ref. 20 and superior to that displayed by the MC1H-PBE approach of that same work.

We note that the quality of the $\lambda$-MCPDFT results, like that of the v2RDM-driven CASSCF, is independent of the $N$-representabilty of the underlying RDMs, in this case. As mentioned above, the likely reason for this behavior is the small size of the active spaces 
employed. Both sets of conditions we consider yield exact CASSCF RDMs for systems with $(2 \mathrm{e}, 2 \mathrm{o})$ active spaces, and the $(4 \mathrm{e}, 4 \mathrm{o})$ active spaces are not so large that we would expect significantly differences to arise between RDMs satisfying the PQG and PQG+T2 conditions. We also note that full translation of the OTPD functionals does not necessarily lead to a reduction in the MAEs associated with MCPDFT or $\lambda$-MCPDFT. For example, a fully-translated PBE functional with a $20 \%$ admixture of nonlocal exchange (the optimal value for this fully-translated functional) performs slightly worse than the corresponding translated functional; the MAE in this case is $2.28 \mathrm{kcal} \mathrm{mol}^{-1}$. A complete set of O3ADD6 relative energies and MAEs for translated and fully-translated MCPDFT and $\lambda$-MCPDFT can be found in the Supporting Information.

\section{CONCLUSIONS}

We have presented a one-parameter global hybrid extension of the multiconfigurational pair density functional theory approach, inspired by the multiconfigurational one-parameter hybrid DFT developed in Ref. 20. Like MC1H, $\lambda$-MCPDFT relies upon a linear decomposition of the electron-electron interaction operator to incorporate a fraction of nonlocal exchange into the MCPDFT formalism. Unlike MC1H, $\lambda$-MCPDFT is not a self-consistent theory, and the functionals we employ are functionals of the on-top pair density, whereas the those employed in Ref. 20 are standard functionals of the spin density. In both of these respects, the present approach is similar to the pCCD- $\lambda$ DFT of Ref. 21, with the principal distinction unique to this work being the source of the reference on-top pair densities. Not surprisingly, the admixture of nonlocal exchange improves the quality of the MCPDFT energy, as measured by NPEs in molecular dissociation curves and the performance of the approach when applied to the O3ADD6 benchmark data set.

The results presented herein represent a best-possible scenario for the accuracy of $\lambda$-MCPDFT, in that the mixing parameter is system dependent and chosen specifically to minimize the error for the case in question. A universally useful $\lambda$-MCPDFT formalism would require a single mixing parameter optimized over a large data set. Alternatively, the accuracy of the approach could be improved, in principle, by choosing a local mixing parameter, [53 57] $f(\mathbf{r})$, as opposed to a constant value $\lambda$, which could be defined in terms of the total density and the OTPD. In addition, the MCPDFT formalism could be futher 
improved by generalizing the OTPD functionals to consider range separation of the coulomb interaction (see Ref. 22, for example) or double hybrization.

Lastly, we highlight one unfortunate formal aspect of $\lambda$-MCPDFT. One of the nice properties of MCPDFT is that, unlike some other MR+DFT methods, it avoids double counting of electron correlation within the active space. However, upon introducing a nonzero exchange mixing parameter, $\lambda$, this property is formally lost in $\lambda$-MCPDFT. The two-cumulant in Eq. 11 introduces some correlation effects within the active space that may also be described by the OTPD functional. Presumably, this double counting is minimized along with the total error in any fitting procedure used to determine $\lambda$, but we cannot formally guarantee that this is the case.

Acknowledgments This material is based upon work supported by the Army Research Office Small Business Technology Transfer (STTR) program under Grants No. W911NF-16C-0124 and W911NF-19-C0048. Mohammad Mostafanejad was supported by a fellowship from The Molecular Sciences Software Institute under NSF grant ACI-1547580.

[1] T. Helgaker, P. Jørgensen, and J. Olsen, Molecular electronic-structure theory (Wiley, 2000).

[2] A. Szabo and N. S. Ostlund, Modern quantum chemistry : introduction to advanced electronic structure theory (Dover Publications, 1996).

[3] S. Ghosh, P. Verma, C. J. Cramer, L. Gagliardi, and D. G. Truhlar, Chemical Reviews 118, 7249 (2018).

[4] G. Li Manni, R. K. Carlson, S. Luo, D. Ma, J. Olsen, D. G. Truhlar, and L. Gagliardi, J. Chem. Theory Comput. 10, 3669 (2014).

[5] B. O. Roos, P. R. Taylor, and P. E. Siegbahn, Chem. Phys. 48, 157 (1980).

[6] B. O. Roos, The Complete Active Space Self-Consistent Field Method and its Applications in Electronic Structure Calculations (John Wiley \& Sons, Inc., 1987), vol. 68, pp. 399-445.

[7] P. Siegbahn, A. Heiberg, B. Roos, and B. Levy, Phys. Scripta 21, 323 (1980).

[8] P. E. M. Siegbahn, J. Almlöf, A. Heiberg, and B. O. Roos, J. Chem. Phys. 74, 2384 (1981).

[9] A. D. Becke, A. Savin, and H. Stoll, Theor. Chem. Acc. 91, 147 (1995).

[10] L. Gagliardi, D. G. Truhlar, G. L. Manni, R. K. Carlson, C. E. Hoyer, and J. L. Bao, Acc. 
Chem. Res. 50, 66 (2017).

[11] M. Mostafanejad and A. E. DePrince, J. Chem. Theory Comput. 15, 290 (2019).

[12] A. J. Cohen, P. Mori-Sánchez, and W. Yang, Science 321, 792 (2008).

[13] A. D. Becke, J. Chem. Phys. 96, 2155 (1992).

[14] A. D. Becke, J. Chem. Phys. 97, 9173 (1992).

[15] A. D. Becke, J. Chem. Phys. 98, 5648 (1993).

[16] A. D. Becke, J. Chem. Phys. 98, 1372 (1993).

[17] A. D. Becke, J. Chem. Phys. 104, 1040 (1996).

[18] G. Giuliani and G. Vignale, Quantum Theory of the Electron Liquid (Cambridge University Press, Cambridge, 2005), ISBN 9780511619915.

[19] K. Sharkas, J. Toulouse, and A. Savin, J. Chem. Phys. 134, 064113 (2011).

[20] K. Sharkas, A. Savin, H. J. A. Jensen, and J. Toulouse, J. Chem. Phys. 137, 044104 (2012).

[21] A. J. Garza, I. W. Bulik, T. M. Henderson, and G. E. Scuseria, J. Chem. Phys. 142, 044109 (2015).

[22] A. J. Garza, I. W. Bulik, T. M. Henderson, and G. E. Scuseria, Phys. Chem. Chem. Phys. 17, $22412(2015)$.

[23] F. Ying, C. Zhou, P. Zheng, J. Luan, P. Su, and W. Wu, Front. Chem. 7, 225 (2019).

[24] J. Toulouse, K. Sharkas, E. Brémond, and C. Adamo, The Journal of Chemical Physics 135, $101102(2011)$.

[25] C. Kalai and J. Toulouse, J. Chem. Phys. 148, 164105 (2018).

[26] K. Husimi, Proceedings of the Physico-Mathematical Society of Japan. 3rd Series 22, 264 (1940).

[27] J. E. Mayer, Phys. Rev. 100, 1579 (1955).

[28] P.-O. Löwdin, Phys. Rev. 97, 1474 (1955).

[29] R. K. Carlson, D. G. Truhlar, and L. Gagliardi, J. Chem. Theory Comput. 11, 4077 (2015).

[30] C. Garrod and J. K. Percus, J. Math. Phys. 5, 1756 (1964).

[31] Z. Zhao, B. J. Braams, M. Fukuda, M. L. Overton, and J. K. Percus, J. Chem. Phys. 120, 2095 (2004).

[32] R. M. Erdahl, Int. J. Quantum Chem. 13, 697 (1978), ISSN 1097-461X.

[33] M. Higuchi and K. Higuchi, Comput. Theor. Chem. 1003, 91 (2013).

[34] See https://github.com/edeprince3/v2rdm_casscf for v2RDM-CASSCF v0.8, a variational 
two-electron reduced-density-matrix-driven approach to complete active space self-consistent field theory (2018).

[35] J. M. Turney, A. C. Simmonett, R. M. Parrish, E. G. Hohenstein, F. A. Evangelista, J. T. Fermann, B. J. Mintz, L. A. Burns, J. J. Wilke, M. L. Abrams, et al., WIRES Comput. Mol. Sci. 2, 556 (2012).

[36] R. Gáspár, Acta Physica Academiae Scientiarum Hungaricae 35, 213 (1974).

[37] J. C. Slater, Phys. Rev. 81, 385 (1951).

[38] S. H. Vosko, L. Wilk, and M. Nusair, Can. J. Phys. 58, 1200 (1980).

[39] J. P. Perdew, K. Burke, and M. Ernzerhof, Phys. Rev. Lett. 77, 3865 (1996).

[40] A. D. Becke, Phys. Rev. A 38, 3098 (1988).

[41] C. Lee, W. Yang, and R. G. Parr, Phys. Rev. B 37, 785 (1988).

[42] Https://github.com/SinaMostafanejad/OpenRDM.

[43] Https://github.com/edeprince3/RDM-inoles.

[44] T. H. Dunning, J. Chem. Phys. 90, 1007 (1989).

[45] J. L. Whitten, J. Chem. Phys. 58, 4496 (1973).

[46] B. I. Dunlap, J. W. D. Connolly, and J. R. Sabin, J. Chem. Phys. 71, 3396 (1979).

[47] F. Weigend, Phys. Chem. Chem. Phys. 4, 4285 (2002).

[48] F. Aquilante, J. Autschbach, R. K. Carlson, L. F. Chibotaru, M. G. Delcey, L. De Vico, I. Fdez. Galván, N. Ferré, L. M. Frutos, L. Gagliardi, et al., J. Comp. Chem. 37, 506 (2016).

[49] N. Forsberg and P.-Å. Malmqvist, Chem. Phys. Lett. 274, 196 (1997).

[50] G. Ghigo, B. O. Roos, and P. Å. Malmqvist, Chem. Phys. Lett. 396, 142 (2004).

[51] S. E. Wheeler, D. H. Ess, and K. N. Houk, J. Phys. Chem. A 112, 1798 (2008).

[52] Y. Zhao, O. Tishchenko, J. R. Gour, W. Li, J. J. Lutz, P. Piecuch, and D. G. Truhlar, J. Phys. Chem. A 113, 5786 (2009).

[53] B. Miehlich, H. Soll, and A. Savin, Mol. Phys. 91, 527 (1997).

[54] J. Gräfenstein and D. Cremer, Mol. Phys. 103, 279 (2005).

[55] J. Gräfenstein and D. Cremer, Chem. Phys. Lett. 316, 569 (2000).

[56] O. V. Gritsenko, R. van Meer, and K. Pernal, Phys. Rev. A 98, 062510 (2018).

[57] O. V. Gritsenko and K. Pernal, J. Chem. Phys. 151, 024111 (2019). 